\title{
Interpersonal Violence
}

\section{Improving Victim Recognition and Treatment}

I nterpersonal violence (IPV), including domestic violence (DV) and childhood physical and sexual abuse, is a common problem in our society. An estimated 6\% to $15 \%$ of women have experienced domestic violence in the past 12 months, with a lifetime prevalence estimated at $28 \%$ to $54 \% .^{1,2}$ Likewise, childhood physical and sexual abuse prevalence rates range from $3 \%$ to $40 \% .^{3,4}$ Interpersonal violence is associated with numerous long-term health effects, both physical and mental, and increased use of health care services. $^{1,5,6}$

Awareness of domestic violence and its consequences has been increasing among physicians. Unfortunately, although most physicians agree that domestic violence is a serious problem, most are still not screening patients routinely for DV. Physicians cite a number of reasons for their failure to screen patients, including feeling they have inadequate time and resources to care for DV victims as well as a lack of confidence in their abilities to recognize victims and intervene. ${ }^{7,8}$ Encouragingly, training in DV, particularly in the past year, is associated with higher screening rates and greater confidence in caring for victims. However, training may need to be ongoing: one emergency department found high rates of detection after a training intervention, but several years later rates had fallen to baseline levels. ${ }^{9}$ Most U.S. medical schools report training students on DV as part of the medical school curricula, but exactly what is being taught at each school is unclear. ${ }^{10}$

Training on DV improves screening rates, but are we teaching the correct things? We know that the most dangerous time for a woman in a violent relationship is when she tries to leave. Traditionally, DV training has taught that key elements of danger assessments are the presence of crescendo violence, threats with a gun or weapon, and the woman's assessment of her immediate and future danger. ${ }^{1,11,12}$ A study in this month's Journal of General Internal Medicine suggests that some of this teaching may not be correct. Nicolaidis et al. conducted qualitative interviews of 30 women who were victims of near-homicide by their intimate partners. ${ }^{13}$ This study was done in conjunction with an 11-city case-control study comparing data on women who had survived a homicide attempt by an intimate partner to those who were in violent relationships but had not had an attempt on their lives. Women from the case-control study were invited to participate in a more detailed open-ended interview, with the goal of examining the women's lives in-depth to identify patterns that might aid clinicians in predicting or preventing homicide. The findings from this study are striking: nearly half of the 30 women participating did not recognize that their lives were in danger. In addition, although nearly all women had experienced either physical abuse or controlling behavior, the spectrum of violence and control varied considerably. Most of the homicide attempts did occur around relationship changes, although often the relationship was ending for reasons other than the violence. Results from this landmark study suggest we should be counseling our IPV patients differently, recognizing that any level of physical violence or controlling behavior can be a prelude to a homicide attempt. In addition, curricula on domestic violence should be revised to reflect these new findings.

Whereas most physicians are aware of IPV in the form of DV and feel it is their responsibility to address it, a very different situation exists for child abuse. Like DV victims, few patients with a history of childhood physical or sexual abuse are likely to spontaneously report this to physicians, but few physicians screen for childhood abuse. ${ }^{5,14}$ In part, physicians may not think about the possibility of childhood abuse since often reviews of psychiatric and medical conditions published in medical journals have not mentioned childhood abuse as a potential causative factor. In this issue, Springer et al. attempt to remedy this situation by providing a comprehensive overview of the available research on the long-term effects of childhood abuse on adult psychiatric and physical health. ${ }^{15}$ In this article, they summarize the available literature on this topic, synthesizing data from psychology, psychiatry, nursing, and sociological journals together with the few available articles in medical journals. This is the first comprehensive summary of this disparate literature, and should serve as a wakeup call to general internists, who are likely to encounter these victims. Although universal screening for childhood abuse would be ideal, at the very least this article should prompt clinicians to ask about childhood abuse regularly in patients with somatic and psychiatric complaints. Although there is a lack of data on whether detection and referral to therapy improves long-term health outcomes, there is evidence that even brief cognitive behavioral interventions are helpful in reducing patient suffering.

What can we, as general internists, take away from these two articles? First, we should be screening our patients universally for both childhood and adult IPV, recognizing that IPV may be an underlying factor in many 
patients with somatic and psychiatric complaints. As Springer et al. remind us, we must be vigilant for childhood abuse as well as adult DV. Second, we must advocate for teaching our medical students, residents, and colleagues about screening and intervening for IPV. We must make sure that we are updating our teaching as new findings, like those of Nicolaidis et al., become evident. Third, collaborations between psychosocial and biomedical researchers on IPV may yield new insights into the prevention and treatment of IPV and we should seek out these collaborations. Fourth, for many clinicians pressed for time, it is easier to order a plethora of diagnostic testing in a patient presenting with nonspecific complaints than to invest the time required to build trust with the patient and elicit a history of IPV. It is easy to become overwhelmed in a busy clinic with limited time for each patient. Insurers and policy makers should recognize the importance of time spent taking detailed histories and counseling patients, and should ensure adequate reimbursement for these services. We must continue to remind our students, our residents, our colleagues, and ourselves that outstanding medical care begins with a trusting doctor-patient relationship. Finally, clinicians cannot be expected to bear the sole responsibility for screening for and treating IPV. Future research should be focused on developing and implementing system changes to improve care for IPV survivors. Such system changes could enhance screening practices and provide linkages to comprehensive treatment and counseling services.-Lorrie Elliott, MD, Section of General Internal Medicine, University of Chicago Medical Center, Chicago, IL.

\section{REFERENCES}

1. Eisenstat S, Bancroft L. Domestic violence. N Eng J Med. 1999:341:886-92.
2. Abbott J, Johnson R, Koziol-McLain J, Lowenstein S. Domestic violence against women: incidence and prevalence in an emergency department population. JAMA. 1995;273:1763-7.

3. MacMillan H, Fleming J, Trocme N, et al. Prevalence of child physical and sexual abuse in the community: results from the Ontario Health Supplement JAMA. 1997;279:131-5.

4. Ernst C, Angst J, Foldenyi M. Sexual abuse in childhood. Frequency and relevance for adult morbidity: data of a longitudinal epidemiological study. Eur Arch Psychiatry Clin Neurosci. 1993;242:293300 .

5. McCauley J, Kern D, Kolodner K, et al. The 'battering syndrome.' Prevalence and clinical characteristics of domestic violence in primary care internal medicine practices. Ann Intern Med. 1995; 123:737-46.

6. Council on Scientific Affairs American Medical Association. Violence against women. Relevance for medical practitioners. JAMA. 1992;267:3184-9.

7. Elliott L, Nerney M, Jones T, Friedmann P. Barriers to screening for domestic violence. J Gen Intern Med. 2002;17:112-6.

8. Rodriguez M, Bauer H, McLoughlin E, Grumbach K. Screening and intervention for intimate partner abuse. Practices and attitudes of primary care physicians. JAMA. 1999;282:468-74.

9. McLeer S, Anwar R, Herman S, Maquiling K. Education is not enough: a systems failure in protecting battered women. Ann Emerg Med. 1989;18:651-3.

10. Alpert E, Tonkin A, Seeherman A, Holtz, A. Family violence curricula in U.S. medical schools. Am J Prev Med. 1998;14:273-82.

11. Alpert E. Violence in intimate relationships and the practicing internist: new 'disease' or new agenda? Ann Intern Med 1995; 123:774-81.

12. Sassetti M. Domestic violence. Primary Care. 1993;20:289-305.

13. Nicolaidis C, Curry MA, Ulrich Y, et al. Could we have known? A qualitative analysis of data from women who survived an attempted homicide by an intimate partner. J Gen Intern Med. 2003;18:78894.

14. Lechner M, Vogel M, Garcia-Shelton L, Leichter J, Steibel K. Selfreported medical problems of adult female survivors or childhood sexual abuse. J Fam Pract. 1993;36:633-8.

15. Springer KW, Sheridan J, Carnes M. The long-term health outcomes of childhood abuse: an overview and a call to action. J Gen Intern Med. 2003;18:863-9. 